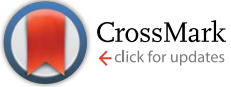

Cite this: RSC Adv., 2017, 7, 1687

Received 7th November 2016 Accepted 4th December 2016

DOI: 10.1039/c6ra26414j

www.rsc.org/advances

\title{
Preparation and characterization of a pH-responsive membrane carrier for meso-tetraphenylsulfonato porphyrin
}

\begin{abstract}
Mingxia Wang, ${ }^{\text {ab }}$ Feng Yan, ${ }^{* a c}$ Lizhi Zhao, ${ }^{\text {ab }}$ Yuzhong Zhang ${ }^{\star a b}$ and Mirco Sorci ${ }^{d}$
A pH-responsive polysulfone-graft-poly(4-vinylpyridine) (PSF-g-P4VP)-blended PSF membrane was prepared for "smart" rejection of meso-tetraphenylsulfonato porphyrin (TPPS) in the feed. The $\mathrm{pH}$ responsive characteristics of the PSF-g-P4VP-blended PSF membranes were determined. The hydraulic permeation results demonstrated that the $\mathrm{pH}$-responsive characteristics behaved as expected, as these were reversible and durable, and occurred with the $\mathrm{pH}$ value switching between 3.0 and 8.0 . Water flux drastically increased from 70 to $1113 \mathrm{~L} \mathrm{~m}^{-2} \mathrm{~h}^{-1}$ at $\mathrm{pH} 8.0$ as the content of PSF-g-P4VP polymer in the blended membrane increased from $10 \%$ to $30 \%$. The static adsorption of TPPS on the $\mathrm{pH}$-responsive blended membrane showed that the adsorption capacity dramatically depended on $\mathrm{pH}$. The $\mathrm{pH}$-sensitive membrane not only plays the role of a smart membrane for rejecting the aggregates of TPPS, but also induces TPPS to assemble on the membrane surface. The flux decreases, whereas the retention of TPPS increases with the decreasing $\mathrm{pH}$. The value of flux was not more than $30 \mathrm{~L} \mathrm{~m}^{-2} \mathrm{~h}^{-1}$ at a pH below 3.0, which provides plenty of time for the self-assembly process of TPPS on the membrane surface. Jaggregates of TPPS were observed on the blended membrane surface below $\mathrm{pH} 2.5$, owing to the inductive effect of cationic P4VP units, whereas J-type aggregates could be only found at $\mathrm{pH} 1.0$ in an aqueous solution.
\end{abstract}

\section{Introduction}

Porphyrin derivatives are well known functional dyes that possess high molar extinction coefficients in the visible region. They have been extensively used as sensors, nonlinear optical materials, energy conversion devices, and solar cells. ${ }^{\mathbf{1 - 4}}$ mesoTetraphenylsulfonato porphyrin (TPPS) is a water-soluble dye and its ability to produce a singlet oxygen $\left({ }^{1} \mathrm{O}_{2}\right)$ is relevant to many biochemical processes, such as in photocatalysis ${ }^{5}$ and in photodynamic therapy to destroy tumors. ${ }^{6}$ There are two types of TPPS aggregates: J-aggregates (edge-to-edge stacking) and $\mathrm{H}$-aggregates (face-to-face stacking). ${ }^{7}$ As illustrated in the literature, ${ }^{8-10}$ the application of TPPS strongly depends on its aggregate forms. Among these two aggregate types, J-aggregates were extensively studied due to their supramolecular chirality ${ }^{\mathbf{1 1}}$

\footnotetext{
${ }^{a}$ State Key Laboratory of Separation Membranes and Membrane Processes, Tianjin Polytechnic University, Tianjin 300387, P. R. China.E-mail: yanfeng.yxm@hotmail. com; zhangyz2004cn@vip.163.com; Fax: +86 22 83955055; Tel: +86 22 8395451; +862283955806

${ }^{b}$ School of Materials Science and Engineering, Tianjin Polytechnic University, Tianjin 300387, P. R. China

${ }^{c}$ School of Environmental and Chemical Engineering, Tianjin Polytechnic University, Tianjin 300387, P. R. China

${ }^{d}$ Department of Chemical and Biological Engineering, Center for Biotechnology and Interdisciplinary Studies, Rensselaer Polytechnic Institute, Troy, NY 12180, USA
}

and photophysical properties for potential applications, such as in optoelectronic devices. ${ }^{\mathbf{2}}$

TPPS aggregation in solution has been extensively investigated. $^{\mathbf{1 3 - 1 6}}$ However, immobilizing porphyrin on the solid carriers is highly desirable for practical use. ${ }^{7,17-19}$ For this reason, a growing body of research is now focusing on the TPPS immobilization on various solid carriers. Balaji et al. prepared an optical sensor using TPPS supported by mesoporous silica for the visual detection of mercury. ${ }^{20}$ Castriciano et al. found that TPPS can be easily anchored on Nafion ${ }^{2}{ }^{21}$ These membranes exhibited interesting optical properties that strongly depended on the extent of TPPS aggregation and the organic solvent used. Our group has studied the adsorption properties and self-aggregation behavior of TPPS on the quaternized polysulfone membranes and found that J-aggregation can also occur on a cationic PSF membrane at $\mathrm{pH} 1.0 .^{22}$ Furthermore, we observed the supramolecular chirality and supramolecular self-assembly of TPPS on an achiral ethylenevinyl alcohol graft poly(2-(dimethylamino)ethyl methylacrylate) membrane. ${ }^{\mathbf{2 3 2 4}}$ Hence, TPPS immobilization on the solid substrates has become a trend for its practical use, and it is believed that a smart membrane $e^{25,26}$ may be a better option for a TPPS carrier than a bead.

$\mathrm{pH}$-responsive smart membranes have attracted great attention due to their potential applications in controlled release, water treatment, tissue engineering, sensors, etc. ${ }^{26,27}$ 
Polymers containing pyridine groups are attractive for preparing $\mathrm{pH}$-responsive functional membranes. ${ }^{28}$ Mika et al. proposed a porous membrane anchored with a poly(4vinylpyridine) (P4VP) $\mathrm{pH}$-sensitive gel. ${ }^{29}$ They found that the water flux reversibly decreased on changing the $\mathrm{pH}$ from 5.5 to 2.6, and the cation rejection sharply increased with the increasing ionization of the incorporated P4VP. Yang et al. presented a poly(ethylene terephthalate)-grafted-P4VP $\mathrm{pH}$ switching membrane. ${ }^{30}$ It was found that the fluxes were close to zero under acidic conditions, whereas they were similar to the blank (unmodified) membranes under neutral conditions. However, to the best of our knowledge, few studies have been focused on the aggregation of TPPS on a porous polymeric carrier, let along on a smart membrane with P4VP brushes that have $\mathrm{p} K_{\mathrm{a}}$ values similar to that of TPPS.

The aim of our work was to fabricate a porous membrane that can "smartly" reject the aggregates of TPPS. Previously, a P4VP graft PSF membrane was synthesized by the surfaceinitiated atom transfer radical polymerization (SI-ATRP) using chloromethylated polysulfone membrane as the initiator. ${ }^{31}$ It was observed that the membrane was fragile after SI-ATRP. Thus, in the present work, we propose a blended membrane of polysulfone-graft-poly(4-vinylpyridine) (PSF-g-P4VP) polymer and PSF polymer. The PSF- $g$-P4VP polymer was prepared via homogeneous ATRP, and the blended membrane was obtained by phase inversion from the mixture of PSF and PSF- $g$-P4VP polymers. The adsorption properties, the rejection performance, and the supramolecular aggregation behavior of TPPS on a $\mathrm{pH}$-responsive blended membrane were studied. Porphyrin immobilization on this $\mathrm{pH}$-responsive membrane could (i) lead to novel applications in smart membranes design, ${ }^{21,32,33}$ (ii) provide additional methods for controlling TPPS aggregation state on the carriers, and (iii) achieve efficient use in the photoelectric and biochemical processes.

\section{Experimental}

\subsection{Materials}

TPPS was purchased from J\&K Scientific Ltd. and used without further purification. Polysulfone (PSF) with a MWCO of $81 \mathrm{kDa}$ was purchased from Dalian Polysulfone Plastic Co. Ltd. (Dalian, China). Chloromethylated polysulfone (CMPSF) was obtained by following a conventional procedure. ${ }^{34} N, N, N^{\prime}, N^{\prime \prime}, N^{\prime \prime}$ Pentamethyl-diethylenetriamine (PMDETA) (98.0\%) and 4vinylpyridine (4VP, 95\%) was purchased from Acros. 4VP was used after vacuum distillation. $\mathrm{CuCl}$ was purified by stirring in $\mathrm{HCl}\left(1 \mathrm{~mol} \mathrm{~L}^{-1}\right)$, filtration, washing with ethanol, and drying under vacuum. All the other chemicals were of analytical grade and used as received.

\subsection{Synthesis of PSF- $g$-P4VP polymer}

PSF- $g$-P4VP polymer was prepared by ATRP. $1 \mathrm{~g}$ of CMPSF was dissolved in $25 \mathrm{~mL} N, N$-dimethylformamide (DMF) in a roundbottom flask at $70{ }^{\circ} \mathrm{C}$. After cooling down to below $5{ }^{\circ} \mathrm{C}, 4 \mathrm{VP}(5$ $\mathrm{mL}$ ), PMDETA (2.3 g), and $\mathrm{CuCl}(0.22 \mathrm{~g})$ were added to the reaction mixture before sealing the flask with a rubber septum.
The reaction mixture was purged with nitrogen for $30 \mathrm{~min}$, and then it was stirred at $60{ }^{\circ} \mathrm{C}$ for $24 \mathrm{~h}$. Finally, the resultant polymer solution was added to the deionized water with stirring and the polymer was precipitated from deionized water. The separated precipitate was redissolved in DMF, and then reprecipitated to remove the impurities. PSF- $g$-P4VP polymer was obtained after filtering, extracting with ethanol in a Soxhlet extractor for $24 \mathrm{~h}$ to eliminate any unreacted 4-vinylpyridine and its autopolymer (P4VP), and drying under vacuum at $60{ }^{\circ} \mathrm{C}$ to a constant weight.

\subsection{Fabrication of the PSF- $g$-P4VP blended PSF membrane}

The PSF- $g$-P4VP was blended with PSF for preparing the membrane via the traditional phase inversion technique. The mixture of PSF- $g$-P4VP and PSF was dissolved in DMF to a concentration of $16 \mathrm{wt} \%$ at $70{ }^{\circ} \mathrm{C}$. PEG 400 acted as a pore forming additive at a concentration of $10 \mathrm{wt} \%$. The casting solution was degassed under vacuum at $25{ }^{\circ} \mathrm{C}$ for $8 \mathrm{~h}$. Then, it was directly spread on a glass plate using a steel knife at a thickness of $200 \mu \mathrm{m}$. After this, the solution films were immediately immersed in a water bath. After complete coagulation, the membrane was peeled off from the support and placed in a fresh deionized water bath for $24 \mathrm{~h}$ to remove any excess solvent. Finally, it was placed in a freeze-dryer to remove water for $24 \mathrm{~h}$ before characterization. Since it was difficult to peel the obtained membrane off the supporting glass at high mass ratios, such as $4: 6$ and $5: 5$, the selected mass ratio of PSF- $g$-P4VP and PSF were $1: 9,2: 8$, and $3: 7$, and the codes of the blended membranes were PSF- $g$-P4VP(10)- $b$-PSF(90), PSF- $g$ $\mathrm{P} 4 \mathrm{VP}(20)-b$-PSF(80), and PSF- $g$-P4VP(30)- $b$-PSF(70), respectively.

\subsection{Water flux measurements}

Water permeation properties of the blended membranes were investigated from $\mathrm{pH} 10.0$ to 2.0 in a "dead-end" filtration apparatus. The $\mathrm{pH}$ values of the solutions with the same concentration of $0.1 \mathrm{~mol} \mathrm{~L} \mathrm{~L}^{-1}$ were adjusted by $\mathrm{Na}_{2} \mathrm{CO}_{3}$, $\mathrm{NaHCO}_{3}, \mathrm{NaAc}, \mathrm{HAc}$, and $\mathrm{HCl}$. Membrane samples were initially compacted at $0.15 \mathrm{MPa}$ with deionized water for $30 \mathrm{~min}$, and then were pretreated by soaking in the test solution for $10 \mathrm{~min}$. Then, water flux was measured with the test solution at $0.10 \mathrm{MPa}$. To observe the flux vs. $\mathrm{pH}$ relationship, all the membranes were first tested with $\mathrm{pH}$ ranging from 10.0 to 2.0, consecutively. Each flux value at a specific $\mathrm{pH}$ for a membrane was the average value of the three readings. The flux was determined by eqn (1):

$$
J_{\mathrm{w}}=\frac{V}{A \Delta t}
$$

where $J_{\mathrm{w}}$ is the water flux $\left[\mathrm{L} \mathrm{m}^{-2} \mathrm{~h}^{-1}\right], V$ is the volume of the test solution permeated $[\mathrm{L}], A$ is the effective membrane area $\left[\mathrm{m}^{2}\right]$, and $\Delta t$ is the permeation time $[\mathrm{h}]$.

\subsection{TPPS static adsorption}

Adsorption properties were measured by immersing the membrane in TPPS solution at $150 \mathrm{rpm}$ (horizontal mechanical 
shaker) and $30{ }^{\circ} \mathrm{C}$. The adsorption capacity was calculated by eqn (2):

$$
Q=\frac{\left(C_{0}-C_{t}\right) V}{m}
$$

where $Q$ is the adsorption capacity $\left[\mathrm{mg} \mathrm{g}^{-1}\right], C_{0}$, and $C_{t}$ are the initial and at time $t$ concentrations of the TPPS solution, respectively $\left[\mathrm{mg} \mathrm{L}^{-1}\right]$, which were determined by UV spectroscopy (Shimadzu UV 2700). ${ }^{23} \mathrm{~m}$ is the dry membrane mass [g] and $V$ is the TPPS solution volume [L]. The influence of $\mathrm{pH}$ on the adsorption properties was investigated: PSF- $g$-P4VP blended PSF membranes (0.04 g) were immersed in TPPS solutions (32 $\left.\mathrm{mL}, C_{0}=60 \mathrm{mg} \mathrm{L}^{-1}\right)$ at different $\mathrm{pH}$ values.

\subsection{TPPS rejection}

TPPS rejection was determined at a given $\mathrm{pH}$ using the deadend filtration experimental setup described in Section 2.4. Permeate and feed solution concentrations were determined by UV spectroscopy, as illustrated in Section 2.5. TPPS rejection, $R_{\text {TPPS }}$ [\%], was calculated by eqn (3):

$$
R=\left(1-\frac{C_{\mathrm{p}}}{C_{\mathrm{f}}}\right) \times 100 \%
$$

where $C_{\mathrm{p}}$ and $C_{\mathrm{f}}$ are the TPPS permeate and feed solution concentrations, respectively $\left[\mathrm{mg} \mathrm{L}^{-1}\right]$.

TPPS aggregates rejected by the membrane surface were studied by a UV-vis spectrophotometer equipped with an integrating sphere.

\subsection{Characterization}

FTIR spectrometer (Bruker, TENSOR37) was used to characterize the chemical composition of the PSF- $g$-P4VP polymer. The ${ }^{1} \mathrm{H}$ NMR spectra of the PSF- $g$-P4VP polymer was obtained using a Bruker DRX-500 NMR instrument (300 MHz). Dimethylsulfoxide- $\mathrm{D}_{6}\left(\right.$ DMSO-D $\left._{6}\right)$ was used as a solvent and tetramethylsilane as an internal standard.

The surface morphology of the membrane was examined using a field emission scanning electron microscope (FE-SEM) (Hitachi S-4800, Japan). The samples were sputtered with gold beforehand and the accelerating voltage was $3.5 \mathrm{kV}$.

\section{Results and discussion}

\subsection{Chemi-physical characterization of the PSF- $g$-P4VP polymer and PSF- $g$-P4VP blended PSF membranes}

The PSF- $g$-P4VP polymer was prepared by ATRP using 4-vinylpyridine as the polymerizable monomer and CMPSF as the initiator, as shown in Scheme 1. The structure of the PSF- $g$-P4VP polymer was characterized by FTIR and ${ }^{1} \mathrm{H}$ NMR.

The FTIR spectra are presented in Fig. 1. In the spectrum of CMPSF (line (a)), several characteristic peaks were identified as follows: (i) the skeletal vibration of the benzene ring (1580 and $1478 \mathrm{~cm}^{-1}$ ); (ii) the asymmetric stretching of the $\mathrm{S}=\mathrm{O}$ bond (1318 and $1291 \mathrm{~cm}^{-1}$ ); (iii) the antisymmetric vibration of C-O-C $\left(1240 \mathrm{~cm}^{-1}\right)$; (iv) the bending vibration of $-\mathrm{CH}_{3}(1382$ $\mathrm{cm}^{-1}$ ); and a weak absorption peak characteristic of $-\mathrm{CH}_{2} \mathrm{Cl}$ $\left(750 \mathrm{~cm}^{-1}\right)$. In the spectrum of PSF- $g$-P4VP polymers (line (b)), the characteristic peak at $1634 \mathrm{~cm}^{-1}$ was attributed to the stretching vibrations of $\mathrm{C}=\mathrm{N}$, confirming the successful polymerization of P4VP on PSF by ATRP. The chemical structures of CMPSF and PSF-g-P4VP were further characterized by ${ }^{1} \mathrm{H}$ NMR. As shown in Fig. 2 line (a), the chemical shifts at 7.83 ppm (peak $\mathrm{b}^{\prime}$ ) and $4.53 \mathrm{ppm}$ (peak $\mathrm{i}^{\prime}$ ) were ascribed to the four protons on the two phenyl rings adjacent to the sulfonyl group and the two protons of $-\mathrm{CH}_{2} \mathrm{Cl}$, respectively. The degree of substitution (DS) was determined as the ratio between the integrated area of peaks at $4.53 \mathrm{ppm}$ and $7.83 \mathrm{ppm}$ in the ${ }^{1} \mathrm{H}$ NMR spectrum. ${ }^{35}$ The DS value of the CMPSF sample was approximately 0.4 , indicating that $40-\mathrm{CH}_{2} \mathrm{Cl}$ groups were grafted for each 100 repeat units. The ${ }^{1} \mathrm{H}$ NMR spectra of PSF- $g$-P4VP are shown in Fig. 2 line (b). The peaks at $2.75 \mathrm{ppm}$ (peak j) and $2.90 \mathrm{ppm}$ (peak k) are ascribed to $-\mathrm{CH}_{2}$ - and in the main chain of P4VP. The chemical shift at $8.25 \mathrm{ppm}$ (peak q) was attributed to the two protons on the pyridine ring closest to the nitrogen atom. The bulk graft concentration or the $[\mathrm{P} 4 \mathrm{VP}] /[\mathrm{CMPSF}]$ molar ratio can be calculated from the ${ }^{1} \mathrm{H}$ NMR spectrum according to the following relationship:

$$
\frac{[\mathrm{P} 4 \mathrm{VP}]}{[\mathrm{CMPSF}]}=\frac{A_{\mathrm{q}} / 2}{\left(A_{\mathrm{b}} / 4\right) \times \mathrm{DS}}
$$

where [CMPSF] and [P4VP] represent the amounts of the PSF unit with a $-\mathrm{CH}_{2} \mathrm{Cl}$ group and the grafted vinylpyridine group, respectively. $A_{\mathrm{q}}$ and $A_{\mathrm{b}}$ are the integrated areas of peak q $(\delta 8.25$ $\mathrm{ppm})$ and peak $\mathrm{b}(\delta 7.83 \mathrm{ppm})$, respectively. The factor 2 accounts for the fact that there are 2 protons closest to the nitrogen atom on each pyridine ring, whereas the factor 4 accounts for the fact that there are 4 protons on the two phenyl rings adjacent to the sulfonyl group per repeat unit of the PSF chain. The degree of substitution of the CMPSF sample is 0.4. According to the ${ }^{1} \mathrm{H}$ NMR spectrum, the $[\mathrm{P} 4 \mathrm{VP}] /[\mathrm{CMPSF}]$ molar ratio is 16 , which means 16 vinylpyridine groups were grafted on each activated PSF unit on an average. In summary, the results obtained from FT-IR and ${ }^{1} \mathrm{H}$ NMR demonstrate that the PSF- $g$-P4VP polymer was successfully synthesized.

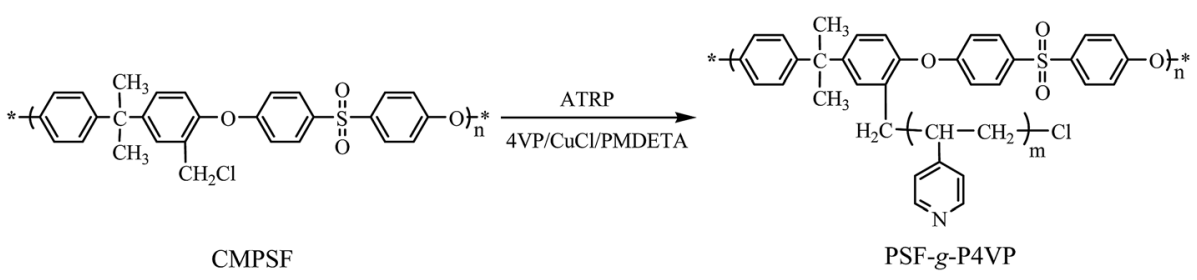

Scheme 1 Synthesis route for PSF-g-P4VP. 


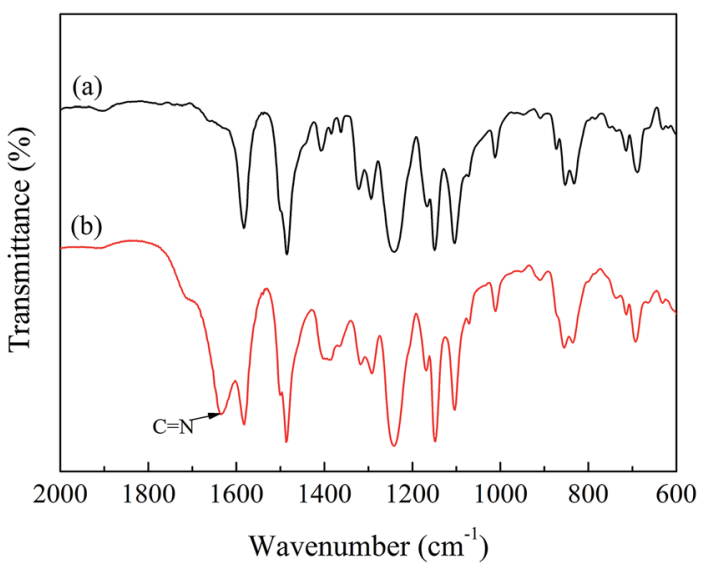

Fig. 1 FT-IR spectra of polymers: (a) CMPSF and (b) PSF-g-P4VP.

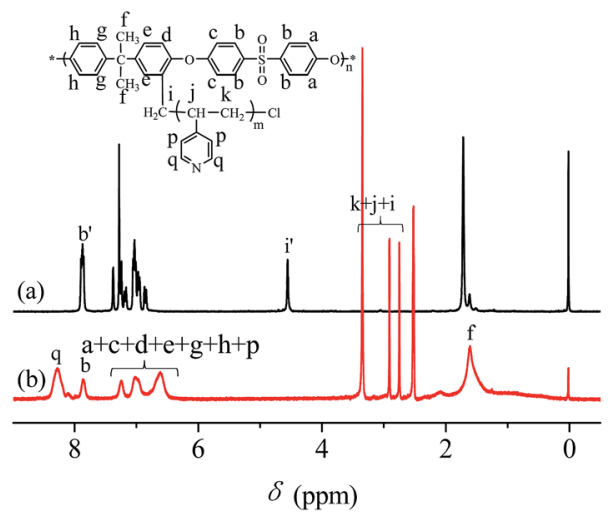

Fig. $2{ }^{1} \mathrm{H}$ NMR spectra: (a) CMPSF in $\mathrm{CDCl}_{3}$ and (b) PSF-g-P4VP in DMSO-D 6 .

The PSF- $g$-P4VP blended PSF membranes were prepared by traditional phase inversion. The obtained membranes were exposed to different $\mathrm{pH}$ solutions for $30 \mathrm{~min}$, quickly rinsed with pure water and rapidly frozen before freeze drying. The morphologies of the blended membrane and PSF blank membrane at $\mathrm{pH} 8.0$ and 2.0 were obtained by FE-SEM, as shown in Fig. 3. Because the PSF blank membrane was not $\mathrm{pH}$ sensitive, there was no morphology difference at $\mathrm{pH} 8.0$ and 2.0. Thus, only the results at $\mathrm{pH} 8.0$ are presented in Fig. 3a-c. As shown in Fig. 3a and b, the surfaces were smooth with the nanometer-sized micropores on the top surface (air side) and micron-sized holes on the bottom surface (glass side). The SEM results of the blended membrane at $\mathrm{pH} 8.0$ are presented in Fig. 3d-f. Similarly, there were nanometer-sized micropores on the top surface of the blended membrane (Fig. 3d). The diameter of the pores (also micron-sized) on the bottom surface (Fig. 3e) was much larger than that of those on the blank PSF membrane (Fig. 3b). However, a very dense surface with many spherical particles could be seen on the top surface of the blended membrane at pH 2.0 (Fig. 3g), and the pore size of the bottom surface at $\mathrm{pH} 2.0$ (Fig. 3h) appeared to be smaller than that at $\mathrm{pH}$ 8.0. Fig. $3 \mathrm{f}$ showed the FE-SEM image of a crosssectional view of the PSF- $g$-P4VP(30)- $b$-PSF(70) membrane at
$\mathrm{pH}$ 8.0. It displayed the typical asymmetric structure with a nearly dense skin layer and finger-like sublayer. The top layer included a honeycomb-like reticulated pore structure, whereas the sublayer showed a fiber-like network, which might provide good permeability. The cross-sectional view at pH 2.0 (Fig. 3i) was similar to that at $\mathrm{pH} 8.0$ (Fig. 3f). The morphologies of the PSF- $g$-P4VP(10)- $b$-PSF(90) and PSF- $g$-P4VP(20)- $b$-PSF(80) membranes (not shown) were similar to that of the PSF- $g$ P4VP(30)- $b$-PSF(70) membrane.

\section{2 pH-responsive permeability of the PSF- $g$-P4VP blended PSF membranes}

P4VP provided the blended membrane with $\mathrm{pH}$-responsivity and the water flux was investigated using ultrafiltration, as shown in Fig. 4. The water flux of the pristine PSF membrane was found below $10 \mathrm{~L} \mathrm{~m}^{-2} \mathrm{~h}^{-1}$ at $0.1 \mathrm{MPa}$ (Fig. 4 line (a)), which indicated that the permeation flux of the PSF membrane was $\mathrm{pH}$-independent. However, the flux of the PSF- $g$-P4VP blended PSF membrane was pH-dependent.

The flux increased after the PSF- $g$-P4VP polymer was mixed with the PSF membrane, which may be ascribed to the hydrophilic pyridine groups. The flux decreased with the increasing concentration of $\mathrm{H}^{+}$, and the most drastic decrease occurred between $\mathrm{pH} 5.0$ and 3.0. The fluxes at $\mathrm{pH}=8.0-10.0$ were always higher than those at $\mathrm{pH}=2.0-3.0$, which could be due to the conformational change of the P4VP chains at the pore surface. At $\mathrm{pH}$ values lower than the $\mathrm{p} K_{\mathrm{a}}$ of $\mathrm{P} 4 \mathrm{VP}$ (about 4.5 ), the pyridine groups were protonated and positively charged; therefore, the P4VP chains were extended due to the repulsion between positive charges, and the membrane gates were in the "closed" state (Fig. 5). Thus, the hydraulic permeability was low. However, under neutral or weakly alkaline conditions, the pyridine groups were deprotonated; therefore, the membrane gates were in the "open" state to reduce the electrostatic repulsions between the P4VP chains on the pore surfaces (Fig. 5). Consequently, the water flux dramatically increased. The abovementioned results could be described by the Hagen-Poiseuille's law, ${ }^{36}$ in which the water flux scales with the fourth power of pore diameter. As abovementioned, the pore diameter was controlled by the $\mathrm{pH}$ value of the solution. Therefore, the permeability was $\mathrm{pH}$-dependent. Moreover, it can be seen from Fig. $4 \mathrm{~b}$ and $\mathrm{d}$ that the PSF- $g$-P4VP blended PSF membrane with higher PSF- $g$-P4VP polymer content in the casting solution resulted in a higher water flux. The water flux increased from 70 to $1113 \mathrm{~L} \mathrm{~m}^{-2} \mathrm{~h}^{-1}$ at $\mathrm{pH} 8.0$ as the content of PSF- $g$-P4VP in the blend membrane increased from $10 \%$ to $30 \%$. This may be due to the increase of P4VP chains on the internal surface of the membrane pores.

To further characterize the $\mathrm{pH}$-responsive performance, a coefficient, called the pH-responsive coefficient $(K),{ }^{37}$ was defined as follows:

$$
K=\frac{J_{\mathrm{pH}=8}}{J_{\mathrm{pH}=3}}
$$

where the numerator and denominator represent the transmembrane fluxes at $\mathrm{pH}=8.0$ and 3.0, respectively. The values 


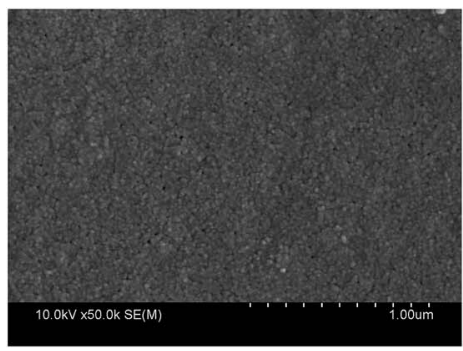

(a)

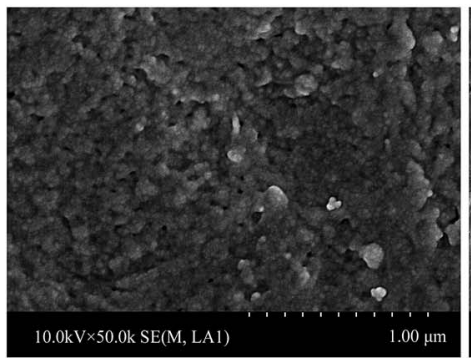

(d)

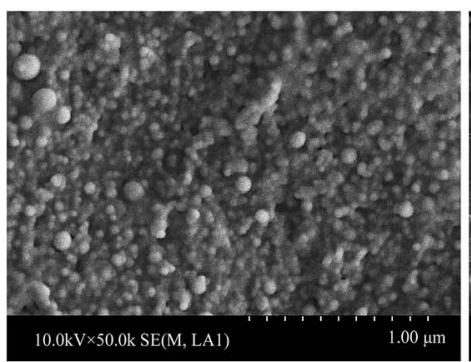

(g)

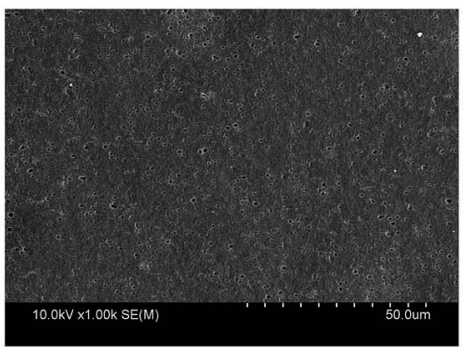

(b)

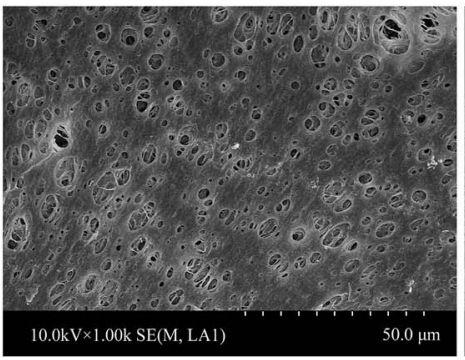

(e)

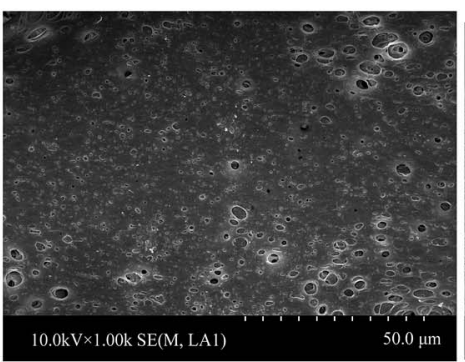

(h)

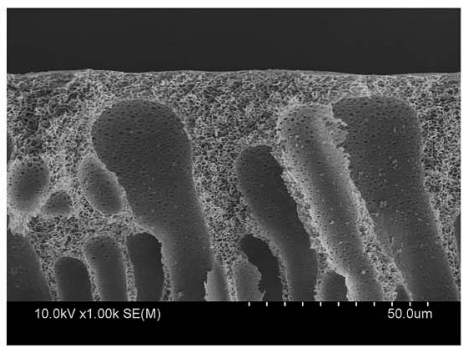

(c)

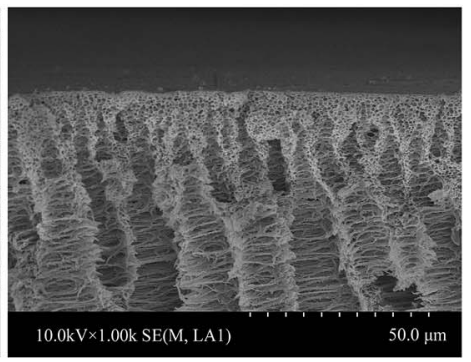

(f)

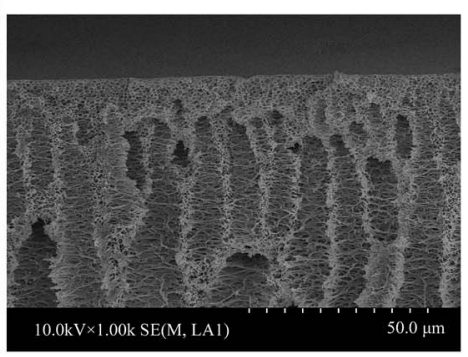

(i)

Fig. 3 FE-SEM images of the PSF-g-P4VP(30)-b-PSF(70) blended membrane and blank PSF membrane: (a)-(c) blank PSF membrane top, bottom, and cross-sectional views at $\mathrm{pH}$ 8.0; (d)-(f) blended membrane top, bottom, and cross-sectional views at pH 8.0; (g)-(i) blended membrane top, bottom, and cross-sectional views at $\mathrm{pH} 2.0$.

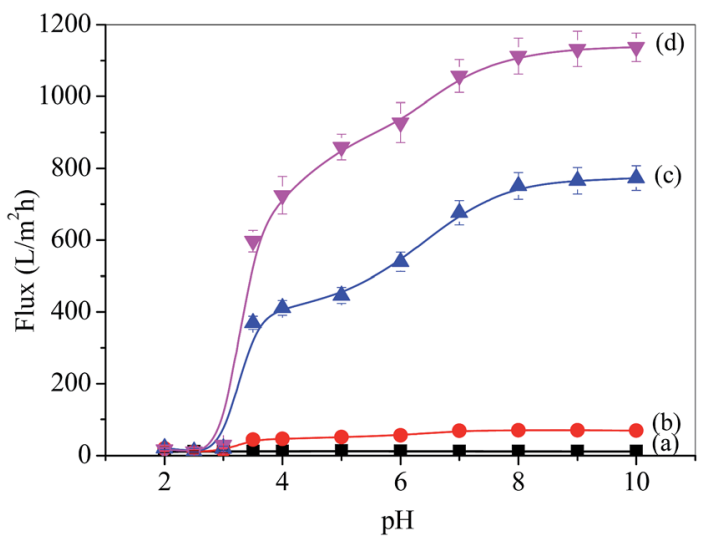

Fig. $4 \mathrm{pH}$-dependence of water permeation through the membranes: (a) PSF, (b) PSF- $g$-P4VP(10)- $b-P S F(90)$, (c) PSF- $g$-P4VP(20)- $b-P S F(80)$, and (d) PSF-g-P4VP(30)- $b-P S F(70)$.

of $K$ for the PSF- $g$-P4VP blended PSF membranes are listed in Table 1, where it could be seen that the $K$-values increased with an increase in the PSF- $g$-P4VP polymer content. The values of $K$
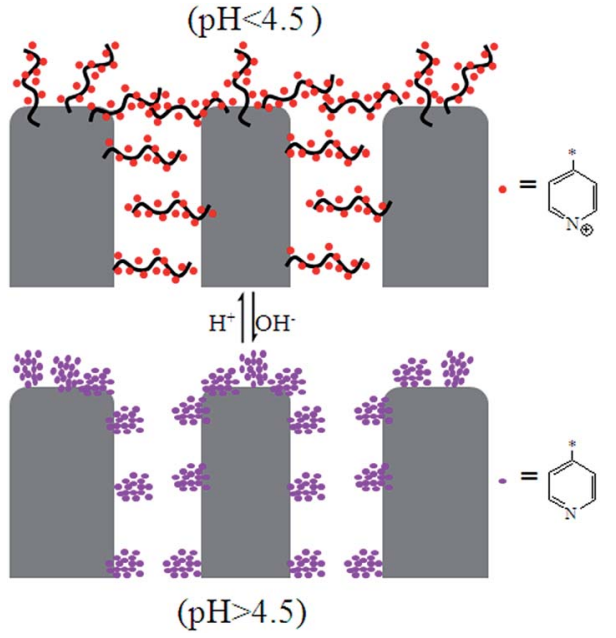

Fig. 5 Schematic for the $\mathrm{pH}$-responsive mechanism of the PSF-gP4VP blended PSF membrane. 
Table 1 The $\mathrm{pH}$-responsive coefficient $(K)$ of the PSF-g-P4VP blended PSF membrane

\begin{tabular}{lllll}
\hline & & \multicolumn{2}{l}{ PSF- $g$-P4VP blended PSF } \\
\cline { 3 - 5 } Membrane & PSF & $\begin{array}{l}\text { PSF- } g \text {-P4VP } \\
10 \%\end{array}$ & $\begin{array}{l}\text { PSF- } g \text {-P4VP } \\
20 \%\end{array}$ & $\begin{array}{l}\text { PSF- } g \text {-P4VP } \\
30 \%\end{array}$ \\
\hline$K$ & 1.0 & 5.1 & 38.1 & 38.5
\end{tabular}

for the blend membranes were much larger than that for the P4VP graft PSF membrane by SI-ATRP, as reported in a previous study (where the values of $K$ varied from 1.5 to 3.0). ${ }^{31}$

The reversibility of water flux was determined by alternately switching the solution $\mathrm{pH}$ between 3.0 and 8.0. The results are shown in Fig. 6, where it can be seen that the permeability immediately changed and was completely reversible. The result indicated that the conformational change of P4VP is reversible.

\subsection{Adsorption of TPPS on the PSF- $g$-P4VP blended PSF membrane}

Static adsorption was performed by immersing a piece of membrane in a TPPS solution. The molecular structure of TPPS is composed of four sulfonate groups (negatively charged), whereas PSF- $g$-P4VP-blended PSF membrane contains blocks of $\mathrm{P} 4 \mathrm{VP}$, which becomes a polycation at a $\mathrm{pH}$ below its $\mathrm{p} K_{\mathrm{a}}$ (around 4.5) ${ }^{38}$ Thus, the electrostatic interaction between the positively charged PSF- $g$-P4VP-blended PSF membrane and the negatively charged TPPS depends on the $\mathrm{pH}$ value of the solution and may regulate the adsorption of TPPS onto the membrane.

Fig. 7 shows the $\mathrm{pH}$-dependent adsorption capacity $(Q)$ of the PSF- $g$-P4VP-blended PSF membranes for TPPS. Thus, the adsorption capacity was relatively small when the $\mathrm{pH}$ of the TPPS solution was above the $\mathrm{p} K_{\mathrm{a}}$ value of the P4VP block, whereas it dramatically increased when the $\mathrm{pH}$ decreased from 5.0 to 3.0 and reached a peak at $\mathrm{pH}$ 2.0. Hence, TPPS efficiently

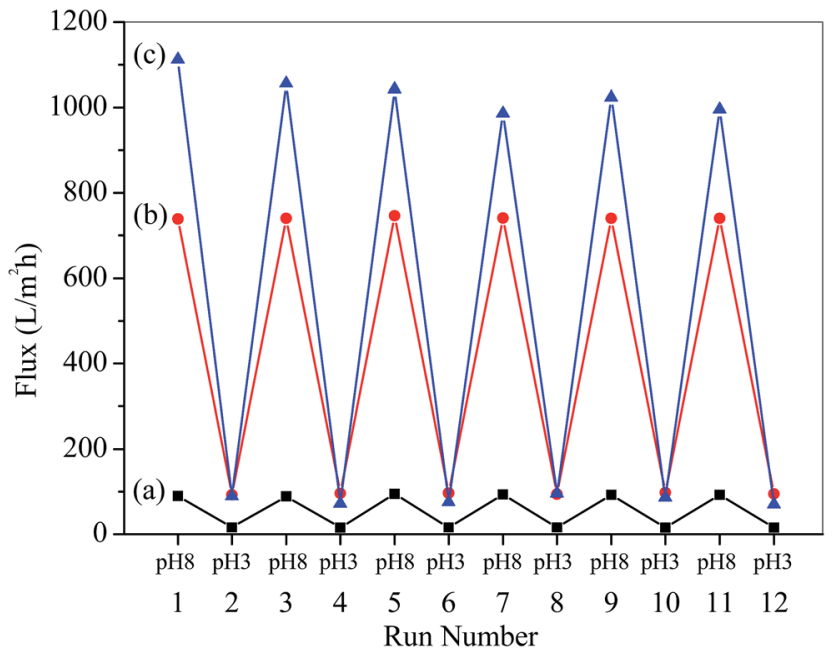

Fig. 6 Reversible changes of water permeation through the membranes as a function of $\mathrm{pH}$ : (a) PSF-g-P4VP(10)- $b-P S F(90)$, (b) PSF- $g$-P4VP(20)- $b-P S F(80)$, and (c) PSF- $g-P 4 V P(30)-b-P S F(70)$.

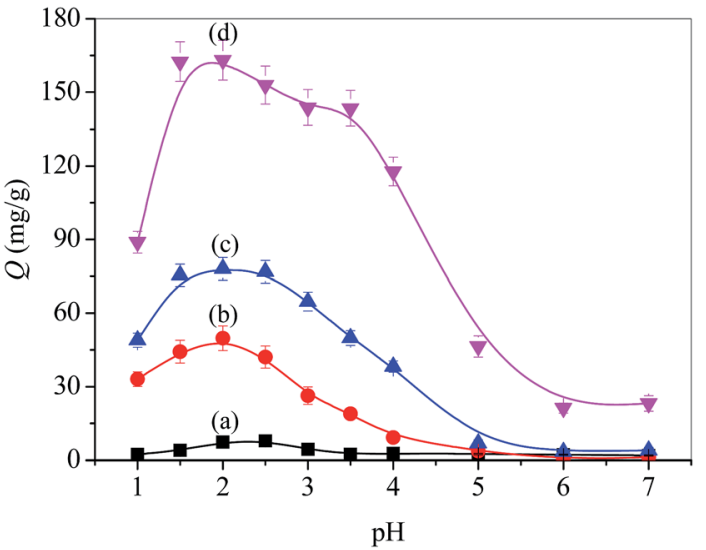

Fig. 7 Effect of $\mathrm{pH}$ on the absorption capacity of the membranes for TPPS: (a) PSF, (b) PSF- $g$-P4VP(10)- $b$-PSF(90), (c) PSF- $g$-P4VP(20)- $b-$ $\operatorname{PSF}(80)$, and (d) PSF-g-P4VP(30)-b-PSF(70). ( $C_{0}=60 \mathrm{mg} \mathrm{L}^{-1}, T=$ $\left.30{ }^{\circ} \mathrm{C}\right)$.

adsorbed on the membrane surface and this was ascribed to the abovementioned typical electrostatic interaction. The adsorption capacity intensely dropped at $\mathrm{pH}$ 2.0-1.0. This may be due to the self-aggregation of the diacid $\mathrm{H}_{4} \mathrm{TPPS}^{2-}$ in the solution, which was unfavorable for adsorption. In addition, it was demonstrated that the adsorption capacity increased with the increasing PSF- $g$-P4VP polymer content in the casting solution for the blended membranes over a wide range of $\mathrm{pH}$ values. In particular, the adsorption capacity increased from 50 to $163 \mathrm{mg}$ $\mathrm{g}^{-1}$ at $\mathrm{pH} 2.0$ as the content of PSF- $g$-P4VP polymer in the blend membrane increased from $10 \%$ to $30 \%$. However, TPPS hardly absorbed on the PSF membrane since both the TPPS and PSF were electrostatically negative and likely repulsed each other. The isoelectric point of PSF is about 3.1. ${ }^{39}$ At a pH higher than 3.1, the membrane surface had an apparent negative charge due to $\mathrm{OH}^{-}$adsorption and repulsion occurs.

\subsection{Rejection of TPPS}

The $\mathrm{p} K_{\mathrm{a}}$ of TPPS is around $4.9{ }^{40}$ which is close to that of P4VP (around 4.5). At a pH lower than 4.5, TPPS and P4VP are protonated (or partially protonated). The former one tends to form aggregates, whereas the smart membrane stretches its P4VP "arms" on the surface and in the pores to capture TPPS and its aggregates via the electrostatic interactions and steric hindrance. As illustrated in Sections 3.2 and 3.3, the PSF-gP4VP(30)- $b$-PSF(70) membrane showed remarkable $\mathrm{pH}^{-}$ responsive permeability and a large amount of adsorption for TPPS. In this section, the retention of TPPS and its aggregates are explored.

The rejection of TPPS was performed using a flow-through method. TPPS solution at a certain $\mathrm{pH}$ was permeated through the PSF- $g$-P4VP(30)-b-PSF(70) membrane. The flux and the rejection were obtained, as shown in Fig. 8. The fluxes of TPPS at $\mathrm{pH} 3.0$ and 2.0 were much lower than that at $\mathrm{pH}$ 8.0. This is due to the closed gates of the membrane, as discussed in Section 3.2, and may also result from the retained aggregates at the gates. However, the rejection for TPPS dramatically 


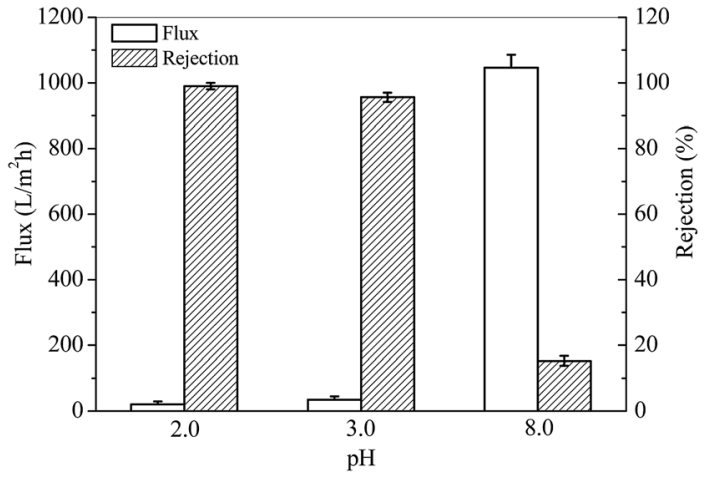

Fig. 8 Flux of the solution and rejection of TPPS at different $\mathrm{pH}$ values.

increased at a pH below the $\mathrm{p} K_{\mathrm{a}}$ value of P4VP. Thus, the pyridine groups were protonated and positively charged at a $\mathrm{pH}$ value lower than 4.5 ( $\mathrm{p} K_{\mathrm{a}}$ of $\left.\mathrm{P} 4 \mathrm{VP}\right)$. The large TPPS rejection is due to (1) the electrostatic adsorption between the positively charged P4VP on the membrane and the negatively charged $\mathrm{SO}_{3}{ }^{-}$of TPPS and (2) the rejection of the TPPS aggregates when the membrane gates were closed at low $\mathrm{pH}$ values. As a result, the rejection for TPPS was $99.0 \%$ at $\mathrm{pH} 2.0$.

\subsection{Aggregation of TPPS on the PSF- $g$-P4VP blended PSF membrane}

In aqueous solution, the existing form of TPPS depends on the pH value. ${ }^{41}$ The monomeric free base $\left(\mathrm{H}_{2}\right.$ TPPS $\left.^{4-}\right)$ translates into a zwitterionic diacid $\mathrm{H}_{4}$ TPPS $^{2-}$ at a $\mathrm{pH}$ below $4.9,{ }^{40}$ which causes changes in the UV-vis absorption spectra. As shown in Fig. 9a line (1), an intense Soret band (413 nm) and four weak Qbands $(633,580,552$, and $515 \mathrm{~nm})$ indicated the presence of the monomeric free base form of TPPS at $\mathrm{pH}$ 7.0. However, the Soret band shifts to $434 \mathrm{~nm}$ and Q-band shifts to $644 \mathrm{~nm}$, illustrating the zwitterionic diacid of TPPS due to the protonation of nitrogen in the macrocycle under acid conditions (Fig. 9a, line (2) at pH 3.0, and line (5) at pH 2.0). Furthermore, a Soret band at $490 \mathrm{~nm}$ and a weak Q-band at $708 \mathrm{~nm}$ in Fig. 9a, line (6) at $\mathrm{pH}$ 1.0 indicated that the J-aggregates of a side-by-side type had formed due to the interaction between the positively charged center of one $\mathrm{H}_{2}$ TPPS $^{4-}$ molecule and the negatively charged peripheral sulfonate groups of the adjacent molecules. ${ }^{42}$

Similarly, the type of TPPS aggregates on the membrane could be controlled by adjusting the $\mathrm{pH}$ value. Fig. 9b shows the UV-visible absorption spectra of TPPS on the PSF- $g$-P4VP(30)- $b$ PSF(70) membrane after the rejection experiments (Section 3.4). The TPPS were mainly in the form of a free base monomer on the blended membrane at a $\mathrm{pH}$ above 2.5. As shown in Fig. 9b curve (2) and curve (3), a Soret band and four Q-bands indicated the existence of the free base monomer of TPPS. The peak positions of TPPS in the aqueous solution and on the PSF- $g$ P4VP- $b$-PSF membrane surface are listed in Table 2 . Note that the Soret band showed a red-shift from $413 \mathrm{~nm}$ to $423 \mathrm{~nm}$ after the TPPS in aqueous solution adsorbed on the membrane surface. In addition, TPPS was found to retain the free base monomer form on the membrane surface at $\mathrm{pH} 3.0\left(<\mathrm{p} K_{\mathrm{a}}\right.$ of TPPS), whereas it normally forms a diacid $\left(\mathrm{H}_{4} \mathrm{TPPS}^{2-}\right)$ in aqueous solution at the same $\mathrm{pH}$ value. Furthermore, a weak shoulder peak at $405 \mathrm{~nm}$ could be seen in Fig. 9b curves (2) and (3) at pH 3.0 and 2.6, respectively. This peak was a characteristic feature of $\mathrm{H}$-dimers of the free base form ${ }^{43}$ and might result from the association of $\pi$-conjugated porphyrin rings. These type of aggregates are supposed to be $\mathrm{H}$-dimers rather than $\mathrm{H}$ oligomers. ${ }^{44}$ This assembly with a small aggregation number was hardly observed by SEM.

A Soret band (488 $\mathrm{nm}$ ) and a weak Q-band (702 nm) was observed in the Fig. 9b spectra (4) to (6), which indicated that the J-aggregated TPPS were obtained on the blended membrane

Table 2 Peak positions of TPPS in aqueous solution and on the membrane surface

\begin{tabular}{llll}
\hline TPPS & $\mathrm{pH}$ & Soret band $(\mathrm{nm})$ & Q-bands $(\mathrm{nm})$ \\
\hline Aqueous solution & 7.0 & 413 & $515,552,580,633$ \\
& 3.0 & 433 & 595,644 \\
Membrane surface & 7.0 & 423 & $519,554,592,647$ \\
& 3.0 & 423 & $519,554,592,647$
\end{tabular}

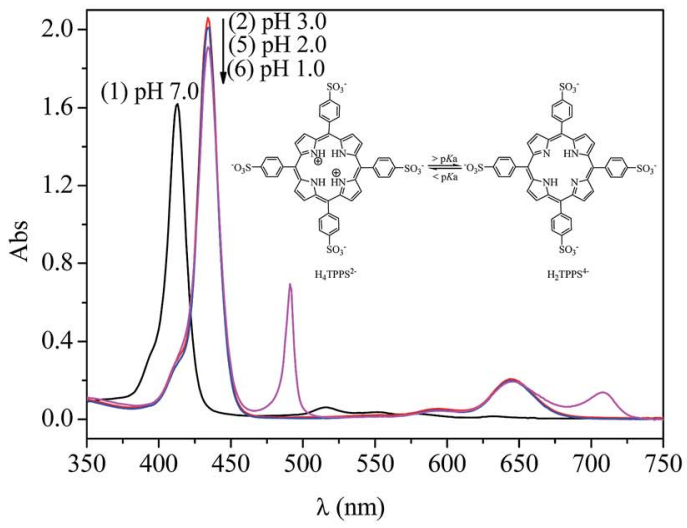

(a)

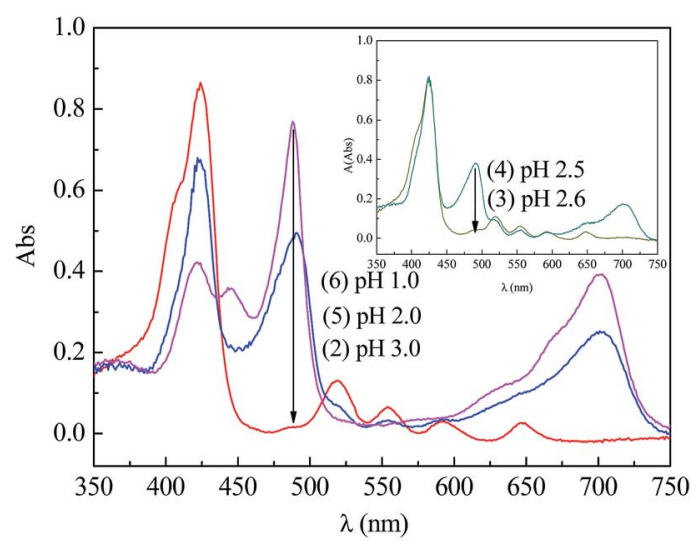

(b)

Fig. 9 UV-vis absorption spectra of TPPS at different pH values: (a) in solution and (b) on PSF-g-P4VP(30)-b-PSF(70) membrane surface. 


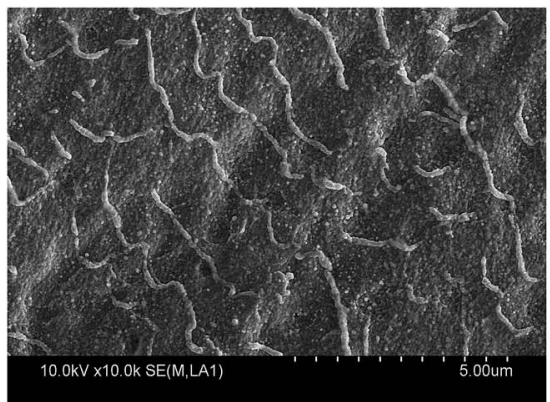

(a)

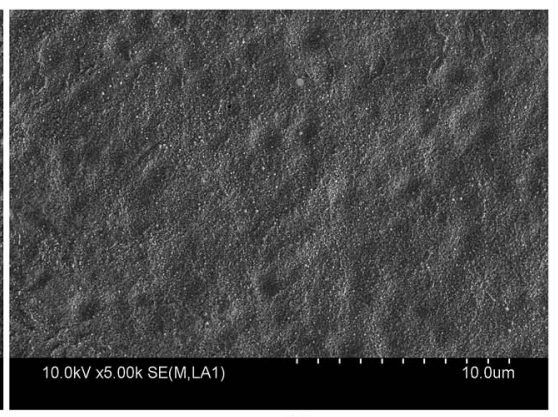

(b)

Fig. 10 FE-SEM images of the PSF-g-P4VP(30)-b-PSF(70) membrane surface at pH 2.0 (a) with TPPS and (b) without TPPS

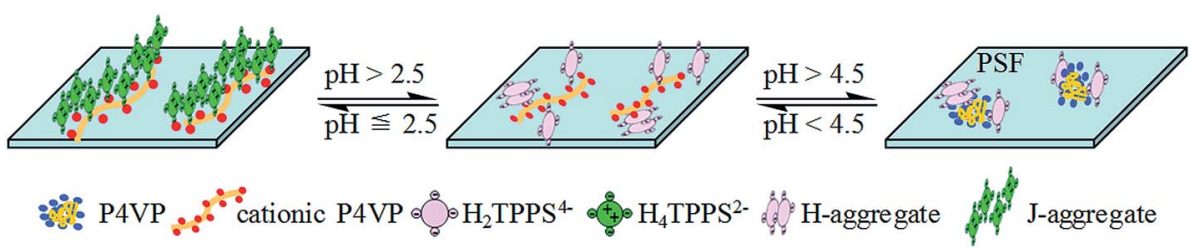

Fig. 11 Schematic for the TPPS aggregates conversion on the PSF-g-P4VP(30)-b-PSF(70) membrane.

at a $\mathrm{pH}$ below 2.5. Furthermore, the intensity of the two bands increased with the decreasing $\mathrm{pH}$, suggesting that the amount of J-aggregation increased. The J-aggregation morphology on the PSF- $g$-P4VP(30)- $b$-PSF(70) membrane surface at $\mathrm{pH} 2.0$ could be clearly seen in Fig. 10a. Relatively linear aggregations with a large aspect ratio, about 50-100 $\mathrm{nm}$ diameter and 2-5 $\mu \mathrm{m}$ length, were aligned on the blended membrane surface. For comparison, the SEM picture of the same membrane at the same pH but without TPPS is also shown in Fig. 10b.

A summary of the conversion of the TPPS aggregation process and phase change from J-type to H-type on the PSF- $g$ P4VP blended PSF membrane by adjusting the $\mathrm{pH}$ is shown in Fig. 11.

\section{Conclusion}

A pH-responsive smart membrane was prepared by blending PSF- $g$-P4VP and PSF through the phase inversion technique. The $\mathrm{pH}$ response of the PSF- $g$-P4VP blended PSF membranes was demonstrated using hydraulic permeation results. The water flux of the blended membrane decreased with the decreasing $\mathrm{pH}$ value, with the most drastic decrease occurring between $\mathrm{pH} 3.0$ and 5.0, and was both reversible and durable with $\mathrm{pH}$ switching between 3.0 and 8.0. At $\mathrm{pH} 3.0\left(<\mathrm{p} K_{\mathrm{a}}\right)$, the membrane pores were in a "closed" state due to the extended conformation of the P4VP chains on the pore surfaces. In contrast, at $\mathrm{pH} 8.0\left(>\mathrm{p} K_{\mathrm{a}}\right)$, the membrane pores were in an "open" state because of the collapsed conformation of the P4VP chains. Moreover, the flux increased with the increasing content of PSF- $g$-P4VP in the casting solution. The PSF- $g$-P4VP blended PSF membranes exhibited a considerably higher adsorption capacity for TPPS than that for the PSF alone. The adsorption capacity was strongly $\mathrm{pH}$-dependent. It increased with the

decrease in the $\mathrm{pH}$ value below the $\mathrm{p} K_{\mathrm{a}}$ of the $\mathrm{P} 4 \mathrm{VP}$ segment and also with the increase of the PSF- $g$-P4VP content in the casting solution. The pH-sensitive membrane acted as a smart membrane to reject TPPS in the solution. At a pH lower than 3.0, the retention was almost $99.0 \%$, which was ascribed to the closed gates of the membrane pores, whereas it was only $15.3 \%$ at $\mathrm{pH} 8.0$ due to the opened gates of the micropores. Furthermore, the low flux and the high retention of TPPS offered a good opportunity for the self-assembly of TPPS. J-type aggregates of TPPS were observed on the PSF- $g$-P4VP blended PSF membrane surface after rejection at a $\mathrm{pH}$ below 2.5, whereas $\mathrm{H}$-type aggregates were found at a $\mathrm{pH}$ above 2.5. The present study provides a valuable insight into the adsorption properties and aggregation behavior of TPPS on a pH-responsive porous membrane that offers a stable carrier for TPPS for use in the electronic devices and biomimetic chemistry.

\section{Conflict of interest}

The authors declare no competing financial interest.

\section{Nomenclature}

Codes

TPPS

PSF

4VP

P4VP

PSF- $g$-P4VP

PSF- $g$-P4VP(10)- $b$ -

PSF(90)
Full name or meaning meso-Tetraphenylsulfonato porphyrin Polysulfone 4-Vinylpyridine Poly(4-vinylpyridine) Polysulfone-graft-poly(4-vinylpyridine) PSF- $g$-P4VP and PSF mass ratio $1: 9$ 


$\begin{array}{ll}\text { PSF- } g \text {-P4VP(20)- } b- & \text { PSF- } g \text {-P4VP and PSF mass ratio } 2: 8 \\ \text { PSF(80) } & \\ \text { PSF- } g \text {-P4VP(30)- } b \text { - } & \text { PSF- } g \text {-P4VP and PSF mass ratio } 3: 7 \\ \text { PSF(70) } & \\ \text { TPPS } & \text { meso-Tetraphenylsulfonato porphyrin } \\ \text { CMPSF } & \text { Chloromethylated polysulfone } \\ \text { ATRP } & \text { Atom transfer radical polymerization } \\ \text { PMDETA } & N, N, N^{\prime}, N^{\prime \prime}, N^{\prime \prime} \text {-Pentamethyl- } \\ & \text { diethylenetriamine } \\ \text { DS } & \text { Degree of substitution } \\ J_{\mathrm{w}} & \text { Water flux } \\ Q & \text { Adsorption capacity } \\ K & \text { pH-responsive coefficient }\end{array}$

\section{Acknowledgements}

The authors gratefully acknowledge the financial support by the National Natural Science Foundation of China (Grant No. 51303130, 51173132, 51373120, 21204064), the Changjiang Scholars and Innovative Research Team in University of Ministry of Education of China (Grant No. IRT13084), the Science and Technology Plans of Tianjin (No. 15PTSYJC00230, 14JCQNJC03900 and 15JCZDJC37600), and China Scholarship Council. The authors would like to thank Professor Georges Belfort in Rensselaer Polytechnic Institute for his assistance in preparing the manuscript.

\section{References}

1 M. B. M. Krishna, N. Venkatramaiah, R. Venkatesan and D. N. Rao, J. Mater. Chem., 2012, 22, 3059-3068.

2 H.-J. Son, S. Jin, S. Patwardhan, S. J. Wezenberg, N. C. Jeong, M. So, C. E. Wilmer, A. A. Sarjeant, G. C. Schatz and R. Q. Snurr, J. Am. Chem. Soc., 2013, 135, 862-869.

3 M. Lv, T. Mei, C. A. Zhang and X. Wang, RSC Adv., 2014, 4, 9261-9270.

4 F. D'Souza and O. Ito, Chem. Soc. Rev., 2012, 41, 86-96.

5 P. Dambruoso, M. Ballestri, C. Ferroni, A. Guerrini, G. Sotgiu, G. Varchi and A. Massi, Green Chem., 2015, 17, 1907-1917.

6 R. Zheng, Z. Wu, Y. Yan, J. Wang and J. Huang, RSC Adv., 2015, 5, 17253-17256.

7 Y. Egawa, R. Hayashida and J.-I. Anzai, Langmuir, 2007, 23, 13146-13150.

8 A. Takahashi and S. Mukamel, J. Chem. Phys., 1995, 103, 7144-7155.

9 C. R. Jeukens, M. C. Lensen, F. J. Wijnen, J. A. Elemans, P. C. Christianen, A. E. Rowan, J. W. Gerritsen, R. J. Nolte and J. C. Maan, Nano Lett., 2004, 4, 1401-1406.

10 A. D. Schwab, D. E. Smith, B. Bond-Watts, D. E. Johnston, J. Hone, A. T. Johnson, J. C. de Paula and W. F. Smith, Nano Lett., 2004, 4, 1261-1265.

11 L. Zhang, J. Yuan and M. Liu, J. Phys. Chem. B, 2003, 107, 12768-12773.

12 A. Miura, Y. Shibata, H. Chosrowjan, N. Mataga and N. Tamai, J. Photochem. Photobiol., A, 2006, 178, 192-200.
13 L. Shen, L. Zhao, R. Qu, F. Huang, H. Gao, Y. An and L. Shi, Nano Res., 2015, 8, 491-501.

14 L. Zhao, R. Ma, J. Li, Y. Li, Y. An and L. Shi, Biomacromolecules, 2008, 9, 2601-2608.

15 Q. Zou, L. Zhang, X. Yan, A. Wang, G. Ma, J. Li, H. Möhwald and S. Mann, Angew. Chem., Int. Ed., 2014, 53, 2366-2370.

16 K. Liu, R. Xing, C. Chen, G. Shen, L. Yan, Q. Zou, G. Ma, H. Möhwald and X. Yan, Angew. Chem., 2015, 127, 510-515.

17 A. Fateeva, P. A. Chater, C. P. Ireland, A. A. Tahir, Y. Z. Khimyak, P. V. Wiper, J. R. Darwent and M. J. Rosseinsky, Angew. Chem., 2012, 124, 7558-7562.

18 E. Collini, C. Ferrante, R. Bozio, A. Lodi and G. Ponterini, J. Mater. Chem., 2006, 16, 1573-1578.

19 Z. Liu, Y. Yi, H. Xu, X. Zhang, T. H. Ngo and M. Smet, Adv. Mater., 2010, 22, 2689-2693.

20 T. Balaji, M. Sasidharan and H. Matsunaga, Analyst, 2005, 130, 1162-1167.

21 M. A. Castriciano, A. Carbone, A. Saccà, M. G. Donato, N. Micali, A. Romeo, G. De Luca and L. M. Scolaro, J. Mater. Chem., 2010, 20, 2882-2886.

22 M. Li, L. Zhao, Y. Zhang, M. Liu, H. Ye, Y. Zhang and X. Chen, Colloid Polym. Sci., 2015, 293, 513-522.

23 L. Zhao, M. Liu, S. Li, A. Li, H. An, H. Ye and Y. Zhang, J. Mater. Chem. C, 2015, 3, 3650-3658.

24 M. Liu, L. Zhao, S. Li, H. Ye, H. An and Y. Zhang, RSC Adv., 2016, 6, 10704-10712.

25 T. Yamaguchi, T. Ito, T. Sato, T. Shinbo and S.-I. Nakao, J. Am. Chem. Soc., 1999, 121, 4078-4079.

26 M. Wang, Q.-F. An, L.-G. Wu, J.-X. Mo and C.-J. Gao, J. Membr. Sci., 2007, 287, 257-263.

27 Z. Zhang, X. Zhu, F. Xu, K. Neoh and E. Kang, J. Membr. Sci., 2009, 342, 300-306.

28 C. Zhao, S. Nie, M. Tang and S. Sun, Prog. Polym. Sci., 2011, 36, 1499-1520.

29 A. M. Mika, R. F. Childs and J. M. Dickson, J. Membr. Sci., 2002, 206, 19-30.

30 B. Yang and W. Yang, J. Membr. Sci., 2005, 258, 133-139.

31 M. Wang, Y. Ji, Y. Zhang, S. Wang, X. Long, L. Zhao and F. Yan, Polym. Mater. Sci. Eng., 2016, 32(3), 64-69.

32 C. A. Steinbeck, M. Ernst, B. H. Meier and B. F. Chmelka, J. Phys. Chem. C, 2008, 112, 2565-2573.

33 S. Jiang and M. Liu, J. Phys. Chem. B, 2004, 108, 2880-2884. 34 L. Li, G. Yan and J. Wu, J. Appl. Polym. Sci., 2009, 111, 19421946.

35 F. Yan, H. Pei, Y. Pei, T. Li, J. Li, B. He, Y. Cheng, Z. Cui, D. Guo and J. Cui, Ind. Eng. Chem. Res., 2015, 54, 3473-3479. 36 Y. M. Lee and J. K. Shim, Polymer, 1997, 38, 1227-1232.

37 T. Luo, S. Lin, R. Xie, X.-J. Ju, Z. Liu, W. Wang, C.-L. Mou, C. Zhao, Q. Chen and L.-Y. Chu, J. Membr. Sci., 2014, 450, 162-173.

38 S. N. Sidorov, L. M. Bronstein, Y. A. Kabachii, P. M. Valetsky, P. L. Soo, D. Maysinger and A. Eisenberg, Langmuir, 2004, 20, 3543-3550.

39 M. Pontié, X. Chasseray, D. Lemordant and J. Lainé, J. Membr. Sci., 1997, 129, 125-133.

40 G. De Luca, A. Romeo and L. M. Scolaro, J. Phys. Chem. B, 2006, 110, 7309-7315. 
41 L. Zhao, X. Wang, Y. Li, R. Ma, Y. An and L. Shi, Macromolecules, 2009, 42, 6253-6260.

42 J. M. Ribó, R. Rubires, Z. El-Hachemi, J.-A. Farrera, L. Campos, G. L. Pakhomov and M. Vendrell, Mater. Sci. Eng., C, 2000, 11, 107-115.
43 N. C. Maiti, S. Mazumdar and N. Periasamy, J. Phys. Chem. B, 1998, 102, 1528-1538.

44 P. Kubát, K. Lang, P. Janda and P. Anzenbacher, Langmuir, 2005, 21, 9714-9720. 